\title{
$S$-Mixing Tuple of Operators on Banach Spaces
}

\author{
Wei Wang, ${ }^{1}$ Yonglu Shu, ${ }^{2}$ and Xingzhong Wang' \\ ${ }^{1}$ College of Mathematics and Statistics, Chongqing University, Chongqing 401331, China \\ ${ }^{2}$ Center of Mathematics, Chongqing University, Chongqing 401331, China
}

Correspondence should be addressed to Wei Wang; wangwei005c@126.com

Received 24 June 2016; Revised 23 August 2016; Accepted 15 September 2016

Academic Editor: Enrique Llorens-Fuster

Copyright (C) 2016 Wei Wang et al. This is an open access article distributed under the Creative Commons Attribution License, which permits unrestricted use, distribution, and reproduction in any medium, provided the original work is properly cited.

We consider the question: what is the appropriate formulation of Godefroy-Shapiro criterion for tuples of operators? We also introduce a new notion about tuples of operators, $S$-mixing, which lies between mixing and weakly mixing. We also obtain a sufficient condition to ensure a tuple of operators to be $S$-mixing. Moreover, we study some new properties of $S$-mixing operators on several concrete Banach spaces.

\section{Introduction}

A continuous linear operator $T$ acting on a topological vector space $X$ is called hypercyclic, if there exists a vector $x \in$ $X$ such that the orbit of $x$ under $T$ is dense in $X$; that is, $\overline{\operatorname{orb}(x, T)}=\overline{\left\{x, T x, T^{2} x, \ldots\right\}}=X$. Such a vector $x$ is called a hypercyclic vector for $T$ and the set of hypercyclic vectors for $T$ will be denoted by $\mathrm{HC}(T)$

Rolewicz [1] and Kitai [2] proved that there does not exist hypercyclic operator on finite-dimensional space. This implies that hypercyclic phenomenon only appears in infinite dimensional space for a single operator. For more details on this topic, one can refer to $[3,4]$.

In 2006, Feldman provided a new class of hypercyclic operators, tuples of operators, and showed us a different direction from the previous results. This gives us hypercyclic phenomenon in both separable infinite dimensional space and finite-dimensional space. The first example that the author showed to us is that there exists an $(n+1)$-tuple of diagonal matrices that is hypercyclic on $\mathbb{C}^{n}$.

For an $m$-tuple of operators we mean a finite sequence of length $m$ of mutually commuting continuous linear operators on a Banach space $X$. Let $\mathscr{T}=\left(T_{1}, T_{2}, \ldots, T_{m}\right)$ be an $m$-tuple acting on a Banach space $X$ (more general complete topology vector space), and $\mathscr{F}$ is the semigroup generated by $T$; that is,

$$
\mathscr{F}=\langle\mathscr{T}\rangle=\left\{T_{1}^{k_{1}} T_{2}^{k_{2}} \cdots T_{m}^{k_{m}}: k_{i} \in \mathbb{N}, i=1, \ldots, m\right\},
$$

which is a finitely generated abelian semigroup.
In $[5,6]$, Feldman extended the definitions of hypercyclic and topologically transitive of single bounded linear operator to tuples of operators. This started a new study in the field of hypercyclicity. In $[7,8]$, Yousefi gave the definition of mixing tuples of operators, and he obtained some sufficient conditions for a tuple of continuous operators to be hereditarily transitive and investigated the relationships between hypercyclicity and $d$-dense orbits of a tuple of operators.

In the past few years, the authors have studied the following dynamical properties of tuples $\mathscr{T}$ :

(1) hypercyclicity: $\overline{\operatorname{orb}(x, \mathscr{T})}=\overline{\{S x: S \in \mathscr{F}\}}=X$;

(2) chaotic (Devaney) [9]: $\mathscr{T}$ is hypercyclic and has a dense set of periodic points;

(3) mixing: for any pair $U, V$ of nonempty open subsets of $X$, there exists some positive integer $N$ such that

$T_{1}^{k_{1}} \cdots T_{m}^{k_{m}}(U) \cap V \neq \varnothing \quad \forall k_{j} \geq N, j=1, \ldots, m ;$

(4) weakly mixing: for a tuple of operators $\mathscr{T}$ and subsets $A, B \subset X$ define the return set as

$$
\begin{aligned}
N_{\mathscr{T}} & (A, B) \\
& =\left\{\left(k_{1}, \ldots, k_{m}\right) \in \mathbb{N}_{0}^{m}: T_{1}^{k_{1}} \cdots T_{m}^{k_{m}}(A) \cap B \neq \varnothing\right\} .
\end{aligned}
$$

Then $\mathscr{T}$ is called weakly mixing if for every quadruple of nonempty open subsets $U_{1}, U_{2}, V_{1}, V_{2} \subset X$ we have that $N_{\mathscr{T}}\left(U_{1}, V_{1}\right) \cap N_{\mathscr{T}}\left(U_{2}, V_{2}\right) \neq \varnothing$. 
From the above results, one can deduce that

$$
\text { mixing } \Longrightarrow \text { weakly mixing } \Longrightarrow \text { hypercyclic. }
$$

Corresponding to Birkhoff's transitive theorem of single continuous map which was proposed by Birkhoff in 1920, Feldman [6] and Yousefi and Ershad [10], respectively, showed that hypercyclicity and topologically transitivity of tuples of operators are equivalent on separable infinite dimension Banach space. Here we describe one of the versions of the extended Birkhoff's transitive theorem as follows.

Theorem 1. Let $X$ be a separable infinite dimensional Banach space $X$ and $\left(T_{1}, T_{2}\right)$ the pair of operators $T_{1}$ and $T_{2}$. The following assertions are equivalent:

(1) $\left(T_{1}, T_{2}\right)$ is hypercyclic;

(2) $\left(T_{1}, T_{2}\right)$ is topologically transitive.

If one of these conditions holds then the set of points in $X$ with dense orbit is a dense $G_{\delta}$-set.

As we know, for a single continuous linear operator, we can use its point spectra and corresponding eigenvalues to judge the dynamical system properties of the operator. In [11], Godefroy and Shapiro gave a criterion. The following theorem we cited was settled by Grosse-Erdmann and Peris in their book [4].

Theorem 2 ((Godefroy-Shapiro criterion) [4, Theorem 3.1]). Let $T$ be an operator. Suppose that the subspaces

$$
\begin{aligned}
X_{0} & :=\operatorname{span}\{x \in X: T x=\lambda x \text { for some } \lambda \\
& \in \mathbb{K} \text { with }|\lambda|<1\}, \\
Y_{0} & :=\operatorname{span}\{x \in X: T x=\lambda x \text { for some } \lambda \\
& \in \mathbb{K} \text { with }|\lambda|>1\}
\end{aligned}
$$

are dense in $X$. Then $T$ is mixing and in particular hypercyclic.

Moreover, if $X$ is a complex space and also the subspace

$$
Z_{0}:=\operatorname{span}\left\{x \in X: T x=e^{\alpha \pi i} x \text { for some } \alpha \in \mathbb{Q}\right\}
$$

is dense in $X$, then $T$ is chaotic.

In our another unpublished paper, we established a generalization version of Godefroy-Shapiro criterion for the tuples of operators. Now we state this result as follows.

Theorem 3. Let $\left(T_{1}, T_{2}\right)$ be a pair of commuting continuous linear operators on a separable infinite Banach space $X$. Suppose that the subspaces

$$
\begin{aligned}
X_{0} & :=\operatorname{span}\left\{x \in X: T_{1} x=\lambda_{1} x, T_{2} x\right. \\
& \left.=\lambda_{2} x \text { for some } \lambda_{1}, \lambda_{2} \in \mathbb{K} \text { with }\left|\lambda_{1}\right|<1,\left|\lambda_{2}\right|<1\right\}, \\
Y_{0} & :=\operatorname{span}\left\{x \in X: T_{1} x=\lambda_{1} x, T_{2} x\right. \\
& \left.=\lambda_{2} x \text { for some } \lambda_{1}, \lambda_{2} \in \mathbb{K} \text { with }\left|\lambda_{1}\right|>1,\left|\lambda_{2}\right|>1\right\}
\end{aligned}
$$

are dense in $X$. Then $\left(T_{1}, T_{2}\right)$ is mixing and in particular hypercyclic.
Moreover, if $X$ is a complex space and also the subspace

$$
\begin{aligned}
Z_{0} & :=\operatorname{span}\left\{x \in X: T_{1} x=\left|\lambda_{1}\right| e^{\alpha \pi i} x, T_{2} x\right. \\
& =\left|\lambda_{2}\right| e^{\beta \pi i} x \text { for some } \lambda_{1}, \lambda_{2} \in \mathbb{K} \text { with }\left|\lambda_{1} \lambda_{2}\right| \\
& =1, \alpha, \beta \in \mathbb{Q}\}
\end{aligned}
$$

is dense in $X$, then $\left(T_{1}, T_{2}\right)$ is chaotic.

Comparing the Godefroy-Shapiro criterion for a single operator and Theorem 3 above, it is natural to try to extend G-S criterion via the product of the joint point spectra of the tuple. However, when considering the product of the joint point spectra that satisfies the conditions in Theorem 3, we find that our idea mentioned above does not work; see Example 9 for the details. Therefore, we turn to consider some special joint point spectra, namely, some special subgroup of the abelian semigroup $\mathscr{F}$ generated by the operator $\mathscr{T}$. To do so, we need to introduce the following definition.

Definition 4. The $m$-tuple $\left(T_{1}, \ldots, T_{m}\right)$ of commuting operators on $X$ is $S$-mixing if for any nonempty open sets $U, V \subset$ $X$, and for any $m$-tuple of sequences of positive integers $\left(k_{1, n}, \ldots, k_{m, n}\right)_{n \in \mathbb{N}}$ such that each sequence $\left(k_{j, n}\right)_{n \in \mathbb{N}}$ is strictly increasing, $j=1, \ldots, m$, and

$$
\sup \left\{\left|k_{i, n}-k_{j, n}\right|: n \in \mathbb{N}, 1 \leq i, j \leq m\right\}<\infty,
$$

there exists $N \in \mathbb{N}$ such that

$$
T^{k_{1, n}} \cdots T^{k_{m, n}}(U) \cap V \neq \varnothing \quad \forall n \geq N .
$$

Remark 5. We will give some examples which are related to the above definition in Section 3. According to the definition of mixing of a single operator $T$, we have that for any nonempty open sets $U, V, N(U, V)$ is cofinite. However, if $\mathscr{T}$ is mixing or $S$-mixing, then $\mathbb{N}_{0}^{2} \backslash N_{\mathscr{T}}(U, V)$ is infinite. Obviously, if $\mathscr{T}$ is mixing, then it must be $S$-mixing, and an $S$-mixing tuple of operators $\mathscr{T}$ must be hypercyclic.

From the definition of $S$-mixing and the remark above, one may ask the following question.

Question 1. Is the S-mixing (for the tuple of operators) stronger than weakly mixing (for the tuple of operators) or is the inverse true?

The following two theorems are our main results in the present paper.

Theorem 6. Let $\left(T_{1}, T_{2}\right)$ be a pair of commuting continuous linear operators on a separable infinite Banach space $X$. Suppose that the subspaces

$$
\begin{aligned}
X_{0} & :=\operatorname{span}\left\{x \in X: T_{1} x=\lambda_{1} x, T_{2} x\right. \\
& \left.=\lambda_{2} x \text { for some } \lambda_{1}, \lambda_{2} \in \mathbb{K} \text { with }\left|\lambda_{1} \lambda_{2}\right|<1\right\}, \\
Y_{0} & :=\operatorname{span}\left\{x \in X: T_{1} x=\lambda_{1} x, T_{2} x\right. \\
& \left.=\lambda_{2} x \text { for some } \lambda_{1}, \lambda_{2} \in \mathbb{K} \text { with }\left|\lambda_{1} \lambda_{2}\right|>1\right\}
\end{aligned}
$$


are dense in $X$. Then $\left(T_{1}, T_{2}\right)$ is $S$-mixing and in particular hypercyclic.

Moreover, if $X$ is a complex space and also the subspace

$$
\begin{aligned}
Z_{0} & :=\operatorname{span}\left\{x \in X: T_{1} x=\left|\lambda_{1}\right| e^{\alpha \pi i} x, T_{2} x\right. \\
& =\left|\lambda_{2}\right| e^{\beta \pi i} x \text { for some } \lambda_{1}, \lambda_{2} \in \mathbb{K} \text { with }\left|\lambda_{1} \lambda_{2}\right| \\
& =1, \alpha, \beta \in \mathbb{Q}\}
\end{aligned}
$$

is dense in $X$, then $\left(T_{1}, T_{2}\right)$ is chaotic.

In the preceding theorem, we consider the product of the joint point spectra to extend the G-S criterion, and it is not hard to find that the pair of operators $\left(T_{1}, T_{2}\right)$ is not mixing. We will give an example (Example 18) below. In the view of preserving the property of mixing, Theorem 3 is more suitable than Theorem 6 as a generalization of G-S criterion for tuples of operators.

The following theorem gives a positive answer to Question 1.

Theorem 7. Let $\left(T_{1}, T_{2}\right)$ be a pair of commuting continuous linear operators on a separable infinite Banach space $X$. If $\left(T_{1}, T_{2}\right)$ is $S$-mixing, then $\left(T_{1}, T_{2}\right)$ must be weakly mixing. However, the inverse is not true.

Based on Theorem 7, we obtain a new notion of a dynamic system between mixing and weakly mixing. Let $\left(\left(T_{1}, T_{2}\right), X\right)$ be a dynamic system; then we have

$$
\begin{aligned}
\operatorname{mixing} & \Longrightarrow S \text {-mixing } \Longrightarrow \text { weakly mixing } \\
& \Longrightarrow \text { hypercyclic. }
\end{aligned}
$$

\section{Proofs of Main Results}

Throughout this paper, let $\mathbb{D}$ denote the unit disk $\mathbb{D}=\{\lambda \in$ $\mathbb{C}:|\lambda|<1\}$ and $\mathbb{T}$ denote the unit circle $\mathbb{T}=\{\lambda \in \mathbb{C}:|\lambda|=1\}$. $H(\mathbb{C})$ denotes the entire function space on $\mathbb{C}$.

Although the proof of Theorem 6 is simple and similar to the proof of single mixing operator, we still give a brief proof for the sake of completeness.

Proof of Theorem 6. Let $U, V$ be a pair of nonempty open subsets of $X$; by the hypothesis, we can find $x \in X_{0} \cap U$ and $y \in Y_{0} \cap V$. Hence these vectors can be expressed in the form

$$
\begin{aligned}
& x=\sum_{j=1}^{m} a_{j} x_{j}, \\
& y=\sum_{j=1}^{m} b_{j} y_{j},
\end{aligned}
$$

where $T_{1} x_{j}=\lambda_{j, 1} x_{j}, T_{2} x_{j}=\lambda_{j, 2} x_{j}, T_{1} y_{j}=\mu_{j, 1} y_{j}$, and $T_{2} y_{j}=$ $\mu_{j, 2} y_{j}$, for certain scalars $a_{j}, b_{j}, \lambda_{j, 1}, \lambda_{j, 2}, \mu_{j, 1}, \mu_{j, 2} \in \mathbb{K}$ with $\left|\hat{\lambda}_{j, 1} \lambda_{j, 2}\right|<1,\left|\mu_{j, 1} \mu_{j, 2}\right|>1, j=1, \ldots, m$.
For any pair of sequences of positive integers $\left(k_{1, n}, k_{2, n}\right)_{n \in \mathbb{N}}$ such that each sequence $\left(k_{j, n}\right)_{n \in \mathbb{N}}$ is strictly increasing, $j=1,2$, and

$$
\begin{aligned}
& \sup \left\{\left|k_{1, n}-k_{2, n}\right|: n \in \mathbb{N}\right\}<\infty, \\
& T_{1}^{k_{1, n}} T_{2}^{k_{2, n}} x=\sum_{j=1}^{m} a_{j} \lambda_{j, 1}^{k_{1, n}} \lambda_{j, 2}^{k_{2, n}} x_{j} \\
& =\sum_{j=1}^{m} a_{j} \lambda_{j, 1}^{k_{1, n}-k_{2, n}}\left(\lambda_{j, 1} \lambda_{j, 2}\right)^{k_{2, n}} x_{j} \longrightarrow 0, \\
& u_{k_{1, n}, k_{2, n}}:=\sum_{j=1}^{m} b_{j} \frac{1}{\mu_{j, 1}^{k_{1, n}} \mu_{j, 2}^{k_{2, n}}} y_{j} \\
& =\sum_{j=1}^{m} b_{j} \frac{1}{\mu_{j, 1}^{k_{1, n}-k_{2, n}}\left(\mu_{j, 1} \mu_{j, 2}\right)^{k_{2, n}}} y_{j} \longrightarrow 0
\end{aligned}
$$

as $n \rightarrow \infty$ and

$$
T_{1}^{k_{1, n}} T_{2}^{k_{2, n}} u_{k_{1}, k_{2}}=\sum_{j=1}^{m} b_{j} y_{j}=y
$$

for all $k_{1, n}, k_{2, n} \geq 0$, then one has

$$
\begin{array}{r}
x+u_{k_{1}, k_{2}} \in U, \\
T_{1}^{k_{1, n}} T_{2}^{k_{2, n}}\left(x+u_{k_{1, n}, k_{2, n}}\right) \in V .
\end{array}
$$

It follows that $\left(T_{1}, T_{2}\right)$ is $S$-mixing and also hypercyclic.

Moreover, if $X$ is a complex space, then iterations of the pair of operators show to us that

$$
\begin{aligned}
T_{1}^{k_{1, n}} T_{2}^{k_{2, n}} x & =\left|\lambda_{1}\right|^{k_{1, n}} e^{k_{1, n} \alpha \pi i}\left|\lambda_{2}\right|^{k_{2, n}} e^{k_{2, n} \beta \pi i} x \\
& =\left|\lambda_{1}\right|^{k_{1, n}}\left|\lambda_{2}\right|^{k_{2, n}} e^{\left(k_{1, n} \alpha+k_{2, n} \beta\right) \pi i} x .
\end{aligned}
$$

Since $\alpha, \beta \in \mathbb{Q}$, there exists $k_{1, n}=k_{2, n}$ such that $T_{1}^{k_{1, n}} T_{2}^{k_{2, n}}=$ $e^{k_{1, n}(\alpha+\beta) \pi i}$. Hence $Z_{0}$ is precisely the set of periodic points of the operator $\left(T_{1}, T_{2}\right)$. Thus $\left(T_{1}, T_{2}\right)$ is chaotic whenever $Z_{0}$ is also dense in $X$. This completes the proof.

Remark 8. For a pair of operators $\left(T_{1}, T_{2}\right)$, if it is $S$-mixing, one considers $k_{2, n}=k_{1, n+1}$ for any $n \in \mathbb{N}$; then the bounded sup condition is exactly the fact that the sequence $\left(k_{1, n}\right)_{n}$ (and thus $\left.\left(k_{2, n}\right)_{n}\right)$ is syndetic. For single operators, the notion of syndetical hypercyclicity was studied in $[12,13]$.

Next, we give an example that satisfies the conditions of Theorem 6, but not mixing.

Example 9. Let $\left(\mu_{1} B, \mu_{2} B\right)$ be a pair of multiple of backward shift operators acting on any space $X=l^{p}(\mathbb{N}), 1 \leq p<\infty$, or $c_{0}(\mathbb{N})$, where $\mu_{1}, \mu_{2} \in \mathbb{C} \backslash\{0\}$ and $\left|\mu_{1} \mu_{2}\right|>1$. Then $\left(\mu_{1} B, \mu_{2} B\right)$ is $S$-mixing and chaotic, but not mixing.

One can easily determine the eigenvectors of $B$ as being the nonzero multiples of the sequence

$$
e_{\lambda}:=\left(\lambda, \lambda^{2}, \lambda^{3}, \ldots\right), \quad|\lambda|<1
$$


with corresponding eigenvalue $\lambda$, and $|\lambda|<1$ ensures that $e_{\lambda} \in X$. Hence, $e_{\lambda}$ is an eigenvector of $\mu_{i} B(i=1,2)$ to the eigenvalue $\mu_{i} \lambda$, and this also ensures that

$$
\begin{aligned}
& \left\{x \in X: \mu_{1} B x=\lambda_{1} x, \mu_{2} B x=\lambda_{2} x \text { for some } \lambda_{1}, \lambda_{2}\right. \\
& \quad \in \mathbb{K}\}
\end{aligned}
$$

is nonempty.

According to [14, Sublemma 7], for any nonempty subset $\Lambda$ of the unit disk $\mathbb{D}$, the set $\operatorname{span}\left\{e_{\lambda} ; \lambda \in \Lambda\right\}$ is dense in $X$. Now we consider the following set:

$$
\begin{aligned}
X_{0} & =\operatorname{span}\left\{x \in X: \mu_{1} B x=\lambda_{1} x, \mu_{2} B x\right. \\
& \left.=\lambda_{2} x \text { for } \lambda_{1}, \lambda_{2} \in \mathbb{K} \text { with }\left|\lambda_{1} \lambda_{2}\right|<1\right\}
\end{aligned}
$$

contains $\operatorname{span}\left\{e_{\lambda}:|\lambda|<1 /\left|\mu_{1} \mu_{2}\right|^{1 / 2}\right\}$. Since $\left|\mu_{1} \mu_{2}\right|>1$ and $|\lambda|<1$, we have

$$
\left\{\lambda \in \mathbb{D}:|\lambda|<\frac{1}{\left|\mu_{1} \mu_{2}\right|^{1 / 2}}\right\}
$$

is a nonempty open subset of $\mathbb{D}$ and thus $X_{0}$ is dense in $X$. Since

$$
\begin{aligned}
Y_{0} & =\operatorname{span}\left\{x \in X: \mu_{1} B x=\lambda_{1} x, \mu_{2} B x\right. \\
& \left.=\lambda_{2} x \text { for } \lambda_{1}, \lambda_{2} \in \mathbb{K} \text { with }\left|\lambda_{1} \lambda_{2}\right|>1\right\}
\end{aligned}
$$

contains $\operatorname{span}\left\{e_{\lambda}: 1 /\left|\mu_{1} \mu_{2}\right|^{1 / 2}<|\lambda|<1\right\}$ and

$$
\begin{aligned}
Z_{0} & =\operatorname{span}\left\{x \in X: \mu_{1} B x=\left|\lambda_{1}\right| e^{\alpha \pi i} x, \mu_{2} B x\right. \\
& =\left|\lambda_{2}\right| e^{\beta \pi i} x \text { for some } \lambda_{1}, \lambda_{2} \in \mathbb{K} \text { with }\left|\lambda_{1} \lambda_{2}\right| \\
& =1, \alpha, \beta \in \mathbb{Q}\}
\end{aligned}
$$

contains $\operatorname{span}\left\{e_{\lambda}:|\lambda|=1 /\left|\mu_{1} \mu_{2}\right|^{1 / 2}\right\}$, one can show that $Y_{0}$ and $Z_{0}$ are both dense in $X$. Therefore, $\left(\mu_{1} B, \mu_{2} B\right)$ is $S$-mixing and chaotic.

Now we are going to show that $\left(\mu_{1} B, \mu_{2} B\right)$ is not mixing. To do this, it sufficient for us to construct a counterexample. By the hypothesis, $\left|\mu_{1} \mu_{2}\right|>1$, let $\left|\mu_{1}\right|=3,\left|\mu_{2}\right|=1 / 2$; there exist large enough positive integers $\alpha, \beta$ such that $3^{\alpha}(1 / 2)^{\beta} \leq 1$. Assume on the contrary that $(3 B,(1 / 2) B)$ is mixing, according to the definition of mixing tuple of operators, for any pair $U, V$ of nonempty open subsets of $X$, there exists some positive integer $N$ such that

$$
\left((3 B)^{k_{1}}\left(\frac{1}{2} B\right)^{k_{2}}\right)(U) \cap V \neq \varnothing
$$

for all $k_{j} \geq N, j=1,2$. Let $\alpha, \beta \geq N$; one has that

$$
\left((3 B)^{\alpha}\left(\frac{1}{2} B\right)^{\beta}\right)(U) \cap V \neq \varnothing .
$$

Then $\left\|(3 B)^{\alpha}((1 / 2) B)^{\beta}\right\|>1$; that is, $3^{\alpha}(1 / 2)^{\beta}>1$; this is a contradiction.
The following corollary follows easily from Theorem 6, which tells us that $S$-mixing is stronger than weakly mixing.

Corollary 10. Let $\left(T_{1}, T_{2}\right)$ be a pair of commuting bounded linear operators on a separable infinite Banach space $X$. If $\left(T_{1}, T_{2}\right)$ satisfies Theorem 6 , then $\left(T_{1}, T_{2}\right)$ must be weakly mixing.

Before giving the proof of Theorem 7, we need another theorem.

Theorem 11. Let $\left(T_{1}, \ldots, T_{m}\right)$ be an $m$-tuple on a separable infinite Banach space $X$. If $\left(T_{1}, \ldots, T_{m}\right)$ is $S$-mixing, then the abelian semigroup $\mathscr{F}$ generated by $\left(T_{1}, \ldots, T_{m}\right)$ contains a mixing operator. Moreover, the $m$-tuple $\left(T_{1}, \ldots, T_{m}\right)$ is $S$ mixing if and only if the operator $T_{1} \cdots T_{m}$ is mixing.

Proof. If $\left(T_{1}, \ldots, T_{m}\right)$ is $S$-mixing, then for any nonempty open subsets $U, V \subset X$ and for any tuple of sequences of positive integers $\left(k_{1, n}, \ldots, k_{m, n}\right)_{n \in \mathbb{N}}$ such that each sequence $\left(k_{j, n}\right)_{n \in \mathbb{N}}$ is strictly increasing, $j=1, \ldots, m$, and

$$
\sup \left\{\left|k_{i, n}-k_{j, n}\right|: n \in \mathbb{N}, 1 \leq i, j \leq m\right\}<\infty,
$$

there exists a positive integer $N$ such that

$$
T_{1}^{k_{1, n}} \cdots T_{m}^{k_{m, n}}(U) \cap V \neq \varnothing
$$

for all $n \geq N$. Take $k_{1, n}=\cdots=k_{m, n}$; then one has

$$
\left(T_{1} \cdots T_{m}\right)^{k_{1, n}}(U) \cap V \neq \varnothing
$$

for all $n \geq N$. This implies that $T_{1} \cdots T_{m}$ is mixing.

Next, we need to show that the operator $T_{1} \cdots T_{m}$ is mixing that implies the $m$-tuple $\left(T_{1}, \ldots, T_{m}\right)$ is $S$-mixing. For any $m$-tuple of sequences of positive integers $\left(k_{1, n}, \ldots, k_{m, n}\right)_{n \in \mathbb{N}}$ such that each sequence $\left(k_{j, n}\right)_{n \in \mathbb{N}}$ is strictly increasing, $j=$ $1, \ldots, m$, and

$$
\sup \left\{\left|k_{i, n}-k_{j, n}\right|: n \in \mathbb{N}, 1 \leq i, j \leq m\right\}<\infty,
$$

we may assume that $k_{1, n} \leq k_{j, n}, j=2, \ldots, m$. Since the operator $T_{1} \cdots T_{m}$ is mixing, for any nonempty open subsets $U, V \subset X$ and for any tuple of sequences of positive integers $\left(k_{1, n}, \ldots, k_{m, n}\right)_{n \in \mathbb{N}}$ above, we have that

$$
\left(T_{1} \cdots T_{m}\right)^{k_{1, n}}(U) \cap\left(T_{2}^{k_{1, n}-k_{2, n}} \cdots T_{m}^{k_{1, n}-k_{m, n}}\right)(V) \neq \varnothing
$$

for all $n \geq N$. Clearly, (31) is equivalent to

$$
U \cap\left(T_{1} \cdots T_{m}\right)^{-k_{1, n}}\left(T_{2}^{k_{1, n}-k_{2, n}} \cdots T_{m}^{k_{1, n}-k_{m, n}}\right)(V) \neq \varnothing
$$

for all $n \geq N$. Since $T_{1}, \ldots, T_{m}$ are mutually commutative operators, we obtain that

$$
U \cap T_{1}^{-k_{1, n}} \cdots T_{m}^{-k_{m, n}}(V) \neq \varnothing
$$

for all $n \geq N$. It follows that

$$
T_{1}^{k_{1, n}} \cdots T_{1}^{k_{m, n}}(U) \cap V \neq \varnothing
$$

for all $n \geq N$. This completes the proof. 
Now we are ready to give the proof of Theorem 7 .

Proof of Theorem 7. According to Theorem 11, the semigroup $\mathscr{F}$ generated by $\left(T_{1}, T_{2}\right)$ contains a mixing operator $\widetilde{S}=T_{1} T_{2}$, for any nonempty open subsets $U, V, U_{1}, U_{2}, V_{1}, V_{2} \subset X$; there exists a positive integer $\widetilde{N}$ such that $\widetilde{S}^{n}(U) \cap V \neq \varnothing$ for any $n \geq \widetilde{N}$.

Let $S=\widetilde{S}^{n_{1}}, n_{1} \geq \widetilde{N}$; then $S$ is a continuous map that commutes with $\left(T_{1}, T_{2}\right)$ and satisfies $S(U) \cap V \neq \varnothing$ for any nonempty open subsets $U, V \subset X$. Then we can find nonempty open sets $U_{1}^{\prime} \subset U_{1}, V_{1}^{\prime} \subset V_{1}$ such that $S\left(U_{1}^{\prime}\right) \subset$ $U_{2}, S\left(V_{1}^{\prime}\right) \subset V_{2}$.

Since $\left(T_{1}, T_{2}\right)$ is $S$-mixing operator, the return set $N\left(U_{1}^{\prime}, V_{1}^{\prime}\right) \neq \varnothing$. Suppose that $\left(k_{1}, k_{2}\right) \in N\left(U_{1}^{\prime}, V_{1}^{\prime}\right)$; then there exists $x \in U_{1}^{\prime}$ with $T_{1}^{k_{1}} T_{2}^{k_{2}} x \in V_{1}^{\prime}$. Therefore,

$$
\begin{gathered}
S x \in U_{2}, \\
T_{1}^{k_{1}} T_{2}^{k_{2}} S x=S T_{1}^{k_{1}} T_{2}^{k_{2}} x \in V_{2},
\end{gathered}
$$

which yields that $\left(k_{1}, k_{2}\right) \in N\left(U_{2}, V_{2}\right)$. It follows that $N\left(U_{1}^{\prime}\right.$, $\left.V_{1}^{\prime}\right) \subset N\left(U_{2}, V_{2}\right)$. As $N\left(U_{1}^{\prime}, V_{1}^{\prime}\right) \subset N\left(U_{1}, V_{1}\right)$, we have that

$$
\varnothing \neq N\left(U_{1}^{\prime}, V_{1}^{\prime}\right) \subset N\left(U_{1}, V_{1}\right) \cap N\left(U_{2}, V_{2}\right) .
$$

Hence $\left(T_{1}, T_{2}\right)$ is weakly mixing. For the other direction, we will present a counterexample in Example 18. Thus we complete the proof.

\section{Applications}

In this section, we will give some applications of Theorems 6 and 11. For the pair of classical operators, we can easily obtain the following three results via Theorem 11.

Proposition 12. Let $\left(\mu_{1} B, \mu_{2} B\right)$ be a pair of multiple backward shift operators acting on any space ${ }^{p}(\mathbb{N})(1 \leq p<\infty) \operatorname{orc}_{0}(\mathbb{N})$, where $\mu_{1}, \mu_{2} \in \mathbb{C} \backslash\{0\}$ and $\mu_{1} \neq \mu_{2}$; then $\left(\mu_{1} B, \mu_{2} B\right)$ is $S$-mixing if and only if $\left|\mu_{1} \mu_{2}\right|>1$.

Proposition 13. Let $\left(T_{a_{1}}, T_{a_{2}}\right): H(\mathbb{C}) \rightarrow H(\mathbb{C})$ be a pair of translation operators, where $T_{a_{i}}: H(\mathbb{C}) \rightarrow H(\mathbb{C}), i=1,2$, defined by $T_{a_{i}} f(z)=f\left(z+a_{i}\right)$, for any $f \in H(\mathbb{C})$. Then $\left(T_{a_{1}}, T_{a_{2}}\right)$ is S-mixing if and only if $a_{1}+a_{2} \neq 0$.

Proposition 14. Let $H^{2}(\mathbb{D})$ be a Hardy-Hilbert space of holomorphic function on the complex unit disk $\mathbb{D}$. Let $\varphi_{i}(i=$ $1,2)$ be a nonconstant bounded holomorphic function, $M_{\varphi_{i}}$ a multiplication operator, and $M_{\varphi_{i}}^{*}$ the corresponding adjoint multiplication operator. Let $\left(M_{\varphi_{1}}^{*}, M_{\varphi_{2}}^{*}\right)$ be a pair of adjoint multiplication operators acting on $H^{2}(\mathbb{D})$; then $\left(M_{\varphi_{1}}^{*}, M_{\varphi_{2}}^{*}\right)$ is $S$-mixing if and only if $\left(\varphi_{1} \varphi_{2}\right)(\mathbb{D}) \cap \mathbb{T} \neq \varnothing$.

Using the G-S criterion (Theorem 2), Godefroy and Shapiro in [11] characterized the hypercyclicity of adjoint multipliers, excluding constant multipliers because their adjoint multiplication operators are multipliers of the identity. In [4], the theorem was settled as follows.
Theorem 15 (see [4, Theorem 4.42]). Let $\varphi$ be a nonconstant bounded holomorphic function on $\mathbb{D}$ and let $M_{\varphi}^{*}$ be the corresponding adjoint multiplier on $H^{2}(\mathbb{D})$. Then the following assertions are equivalent:

(1) $M_{\varphi}^{*}$ is hypercyclic;

(2) $M_{\varphi}^{*}$ is mixing;

(3) $M_{\varphi}^{*}$ is chaotic;

(4) $\varphi(\mathbb{D}) \cap \mathbb{T} \neq \varnothing$.

Feldman in [15] focused on pair or tuple of adjoint multiplication operators on the Hardy-Hilbert space; he showed that most pairs of coanalytic Toeplitz operators are hypercyclic. More precisely, the author proved the following theorem.

Theorem 16 (see [15, Theorem 3.1]). Let $f, g \in H^{\infty}(\mathbb{D}) \backslash\{0\}$ satisfy $|f(z)|>1$ for all $z \in \mathbb{D}$ and $|g(z)|>1$ for all $z \in \mathbb{D}$, and let $M_{f}$ and $M_{g}$ be the corresponding multiplication operators on $H^{2}(\mathbb{D})$. Then neither $M_{f}^{*}$ nor $M_{g}^{*}$ is hypercyclic on $H^{2}(\mathbb{D})$, but the following assertions are equivalent:

(1) the pair $\left(M_{f}^{*}, M_{g}^{*}\right)$ is hypercyclic on $H^{2}(\mathbb{D})$;

(2) the semigroup $\mathscr{F}$ generated by the pair $\left(M_{f}^{*}, M_{g}^{*}\right)$ contains a hypercyclic operator;

(3) there exist $n, k \in \mathbb{N}$ such that $f^{n} g^{k}$ is nonconstant and $f^{n} g^{k}(\mathbb{D}) \cap \mathbb{T} \neq \varnothing ;$

(4) there does not exist $p>0$ and $\theta \in \mathbb{R}$ such that $f(z)=$ $e^{i \theta} / g^{p}(z)$ for all $z \in \mathbb{D}$.

Motivated by Theorems 15 and 16, we deduce the following corollary from Theorems 6 and 11 .

Corollary 17. Let $H^{2}(\mathbb{D})$ be a Hardy-Hilbert space of holomorphic function on the complex unit disk $\mathbb{D}$. Let $\varphi_{i}(i=$ $1, \ldots, m)$ be a nonconstant bounded holomorphic function, $M_{\varphi_{i}}$ a multiplication operator, and $M_{\varphi_{i}}^{*}$ the corresponding adjoint multiplication operator. Let $\left(M_{\varphi_{1}}^{*}, \ldots, M_{\varphi_{m}}^{*}\right)$ be a tuple of adjoint multiplication operators acting on $H^{2}(\mathbb{D})$; the following assertions are equivalent:

(1) $\left(M_{\varphi_{1}}^{*}, \ldots, M_{\varphi_{m}}^{*}\right)$ is S-mixing;

(2) $M_{\varphi_{1}}^{*} \cdots M_{\varphi_{m}}^{*}$ is mixing;

(3) $M_{\varphi_{1}}^{*} \cdots M_{\varphi_{m}}^{*}$ is chaotic;

(4) $\left(\varphi_{1} \cdots \varphi_{m}\right)(\mathbb{D}) \cap \mathbb{T} \neq \varnothing$.

Next, we give an example to show that a pair of commutative operators is weakly mixing, but not $S$-mixing.

Example 18. Let $(2 B,(1 / 3) B)$ be a pair of multiple backward shift operators acting on any space $l^{p}(\mathbb{N}), 1 \leq p<\infty$, or $c_{0}(\mathbb{N})$, and then $(2 B,(1 / 3) B)$ is hypercyclic also weakly mixing, but not $S$-mixing. 
Assume on the contrary that $(2 B,(1 / 3) B)$ is $S$-mixing; then for any nonempty open subsets $U, V \subset X$,

$$
2^{k_{1}} \frac{1}{3^{k_{2}}} B^{k_{1}+k_{2}}(U) \cap V \neq \varnothing \quad \text { for } \sup _{k_{1}, k_{2} \geq N}\left|k_{1}-k_{2}\right|<\infty .
$$

Take $m_{1}=m_{2} \geq N$; we have

$$
2^{m_{1}} \frac{1}{3^{m_{2}}} B^{m_{1}+m_{2}}(U) \cap V \neq \varnothing
$$

Equivalently,

$$
\left(\frac{2}{3} B^{2}\right)^{m_{2}}(U) \cap V \neq \varnothing \quad \forall m_{2} \geq N
$$

Hence the operator $(2 / 3) B^{2}$ is mixing, but this is a contradiction.

Next, we check that the operator $(2 B,(1 / 3) B)$ satisfies hypercyclic criterion of tuple of operators [6, Proposition 2.5]. We know that the finite sequences (i.e., sequences of the form $\left.\left(x_{1}, \ldots, x_{n}, 0,0, \ldots\right), n \geq 1\right)$ constitute a dense subset of $X$.

Let $X_{0}$ be the finite sequences, and let $\left(n_{j}\right)_{j \geq 1},\left(k_{j}\right)_{j \geq 1}$ be increasing sequences of positive integers and satisfy $\left(n_{j}-\right.$ $\left.k_{j}\right) \rightarrow+\infty$ as $j \rightarrow \infty$. Let $S_{j}: X_{0} \rightarrow X_{0}$, defined by $S_{j}=$ $(1 / 2)^{n_{j}} 3^{k_{j}} F^{n_{j}+k_{j}}$; then for any $x \in X_{0},(2 B)^{n_{j}}((1 / 3) B)^{k_{j}} x \rightarrow 0$, $(2 B)^{n_{j}}((1 / 3) B)^{k_{j}} S_{j} \rightarrow I$,

$$
\left\|S_{j} x\right\|=\left(\frac{1}{2} F\right)^{n_{j}}(3 F)^{k_{j}} x \leq \frac{1}{2^{n_{j}}} 3^{k_{j}}\|x\| \longrightarrow 0
$$

as $j \rightarrow \infty$. Hence $(2 B,(1 / 3) B)$ is weakly mixing and in particular hypercyclic. Therefore, $(2 B,(1 / 3) B)$ is hypercyclic and weakly mixing, but not $S$-mixing.

Proposition 19. Under the same assumptions in Proposition 12, if $\left(\mu_{1} B, \mu_{2} B\right)$ is $S$-mixing, then the semigroup $\mathscr{F}$ generated by $\left(\mu_{1} B, \mu_{2} B\right)$ contains a mixing operator. But, the inverse is not true.

Proof. Since $\left(\mu_{1} B, \mu_{2} B\right)$ is an $S$-mixing operator, according to Proposition 12, we have that $\left|\mu_{1} \mu_{2}\right|>1$ and $\mu_{1} \mu_{2} B^{2}$ is a mixing operator. Hence the semigroup $\mathscr{F}$ generated by $\left(\mu_{1} B, \mu_{2} B\right)$ contains a mixing operator. Based on Example 18, we can find that the inverse of this is not true. Thus we complete the proof.

Remark 20. There are examples of tuples $\left(T_{1}, T_{2}\right)$ in which every single operator $T_{1}^{n} T_{2}^{m}$ with $n, m \geq 0, n+m>0$, is mixing (thus the tuple is $S$-mixing), but the corresponding semigroup generated by the tuple $\left(T_{1}, T_{2}\right)$ is not mixing. Consider the example of translation semigroup on a sector of the complex plane given in Example 4.15 in [16]. The subsemigroup generated by the tuple of translations $\left(T_{1}, T_{2}\right)$ of the translation semigroup in this example, where $T_{1}=T_{1+i}$ and $T_{2}=T_{1-i}$, is an example of this type, if we follow the argument given there.

For single operator, the relationships between mixing operators and chaotic operators have been studied very clearly; next we give a brief discussion on the relationships between $S$-mixing tuples and chaotic tuples. Firstly, we present a proposition on the pair of multiple backward shift operators.

Proposition 21. Under the same assumptions in Proposition 12, then the following assertions are equivalent:

(1) $\left(\mu_{1} B, \mu_{2} B\right)$ is chaotic;

(2) $\left(\mu_{1} B, \mu_{2} B\right)$ is hypercyclic;

(3) $N(U, V) \neq \varnothing$ for any nonempty open subsets $U, V \subset X$;

(4) $\max \left\{\left|\mu_{1}\right|,\left|\mu_{2}\right|\right\}>1$;

(5) the semigroup $\mathscr{F}$ generated by $\left(\mu_{1} B, \mu_{2} B\right)$ contains $a$ hypercyclic operator;

(6) the semigroup $\mathscr{F}$ generated by $\left(\mu_{1} B, \mu_{2} B\right)$ contains a chaotic operator;

(7) $\left(\mu_{1} B, \mu_{2} B\right)$ has a dense set of periodic points and $N(U, V) \neq \varnothing$ for any pair of nonempty open subsets $U, V \subset X$.

Proof. (1) $\Rightarrow(2),(2) \Leftrightarrow(3)$, and (5) $\Rightarrow(2)$ are trivial.

(2) $\Rightarrow$ (4): by hypothesis, $\left(\mu_{1} B, \mu_{2} B\right)$ is hypercyclic. According to Theorem 1 , we have that the set $\operatorname{HC}\left(\left(\mu_{1} B, \mu_{2} B\right)\right)$ of all hypercyclic vectors of $\left(\mu_{1} B, \mu_{2} B\right)$ is a dense $G_{\delta}$-set in $X$. Let $\mathscr{B}_{X}=\{x \in X:\|x\|<1\}$; we have

$$
\mathscr{B}_{X} \cap \mathrm{HC}\left(\left(\mu_{1} B, \mu_{2} B\right)\right) \neq \varnothing .
$$

Then there exists some $y \in \mathscr{B}_{X} \cap \mathrm{HC}\left(\left(\mu_{1} B, \mu_{2} B\right)\right)$ such that $\operatorname{orb}\left(\left(\mu_{1} B, \mu_{2} B\right), y\right)$ is dense in $X$. Assume on the contrary that $\max \left\{\left|\mu_{1}\right|,\left|\mu_{2}\right|\right\} \leq 1$; then we have

$$
\operatorname{orb}\left(\left(\mu_{1} B, \mu_{2} B\right), y\right) \subseteq \mathscr{B}_{X} \text {, }
$$

because

$$
\begin{aligned}
\left\|\left(\mu_{1} B\right)^{k_{1}}\left(\mu_{2} B\right)^{k_{2}} y\right\| & \leq\left\|\mu_{1} B\right\|^{k_{1}}\left\|\mu_{2} B\right\|^{k_{2}}\|y\| \\
& \leq\left|\mu_{1}\right|^{k_{1}}\left|\mu_{2}\right|^{k_{2}}\|y\|<1,
\end{aligned}
$$

for all $k_{1}, k_{2} \in \mathbb{N}$. This is a contradiction. Therefore, $\max \left\{\left|\mu_{1}\right|\right.$, $\left.\left|\mu_{2}\right|\right\}>1$.

$(4) \Rightarrow$ (5): Without loss of generality, we may assume that $\left|\mu_{1}\right|>1$, then $\mu_{1} B$ is hypercyclic. Hence the semigroup $\mathscr{F}$ generated by $\left(\mu_{1} B, \mu_{2} B\right)$ contains a hypercyclic operator. Therefore, we get

$$
(2) \Longleftrightarrow(3) \Longrightarrow(4) \Longrightarrow(5) \Longrightarrow(2) \text {. }
$$

Based on the proof above, we can easily obtain $(1) \Rightarrow(4)$.

$(4) \Rightarrow(6)$ : without loss of generality, we assume that $\left|\mu_{1}\right|>1$; then $\mu_{1} B$ is a chaotic operator. Therefore, the semigroup $\mathscr{F}$ generated by $\left(\mu_{1} B, \mu_{2} B\right)$ contains a chaotic operator.

$(6) \Rightarrow(1)$ : by hypothesis, the semigroup $\mathscr{F}$ generated by $\left(\mu_{1} B, \mu_{2} B\right)$ contains a chaotic operator $T$. Let $T=$ $\mu_{1}^{\alpha_{1}} \mu_{2}^{\alpha_{2}} B^{\alpha_{1}+\alpha_{2}}, \alpha_{1}, \alpha_{2} \in \mathbb{N}$; then $T$ has a dense set of periodic points and there exists $x \in X$ such that $\operatorname{orb}(x, T)$ is dense in 
$X$. For any $x \in \operatorname{Per}(T)$, there exists a positive integer $N$ such that $T^{N} x=x$; that is,

$$
\mu_{1}^{\alpha_{1} N} \mu_{2}^{\alpha_{2} N} B^{\alpha_{1} N+\alpha_{2} N} x=x
$$

Hence $x \in \operatorname{Per}\left(\left(\mu_{1} B, \mu_{2} B\right)\right)$; then we have

$$
\begin{gathered}
\operatorname{Per}(T) \subseteq \operatorname{Per}\left(\left(\mu_{1} B, \mu_{2} B\right)\right), \\
\operatorname{orb}(x, T) \subseteq \operatorname{orb}\left(\left(\mu_{1} B, \mu_{2} B\right), x\right) .
\end{gathered}
$$

Therefore, $\left(\mu_{1} B, \mu_{2} B\right)$ has a dense set of periodic points and $\operatorname{orb}\left(\left(\mu_{1} B, \mu_{2} B\right), x\right)$ is dense in $X$. Hence $\left(\mu_{1} B, \mu_{2} B\right)$ is chaotic.

Since $(7) \Leftrightarrow(1)$, we have

$$
(1) \Longrightarrow(4) \Longrightarrow(6) \Longrightarrow(1) \Longleftrightarrow(7) \text {. }
$$

Thus we complete the proof.

Remark 22. Based on Propositions 12 and 21, we can see that the operator $(2 B,(1 / 3) B)$ in Example 18 is chaotic, but not $S$ mixing.

\section{Competing Interests}

The authors declare that they have no competing interests.

\section{Acknowledgments}

The authors are grateful to Dr. Xianfeng Zhao for many discussions. This work was partially supported by a NSFC Grant (11271387), Chongqing Natural Science Foundation (cstc 2013jjB0050), and Project in Chongqing (1020709520130059).

\section{References}

[1] S. Rolewicz, "On orbits of elements," Studia Mathematica, vol. 32, no. 1, pp. 17-22, 1969.

[2] C. Kitai, Invariant closed sets for linear operators [Ph.D. thesis], University of Toronto (Canada), ProQuest LLC, Ann Arbor, Miss, USA, 1982.

[3] F. Bayart and E. Matheron, Dynamics of Linear Operators, vol. 179 of Cambridge Tracts in Mathematics, Cambridge University Press, Cambridge, Uk, 2009.

[4] K.-G. Grosse-Erdmann and A. Peris, Linear Chaos, Universitext, Springer, London, UK, 2011.

[5] N. S. Feldman, "Hypercyclic tuples of operators", in Proceedings of the Mini-Workshop: Hypercyclicity and Linear Chaos, MiniWorkshop, pp. 13-19, August 2006, Oberwolfach Report no. 3, 2254-2256, 2006.

[6] N.S. Feldman, "Hypercyclic tuples of operators and somewhere dense orbits," Journal of Mathematical Analysis and Applications, vol. 346, no. 1, pp. 82-98, 2008.

[7] B. Yousefi, "Hereditarily transitive tuples," Rendiconti del Circolo Matematico di Palermo. Second Series, vol. 60, no. 3, pp. 463467, 2011.

[8] B. Yousefi and J. Izadi, "Para-chaotic tuples of operators," Australian Journal of Mathematical Analysis and Applications, vol. 9, no. 1, article 20, p. 8, 2012.
[9] R. Devaney, Chaotic Dynamical Systems, Addison-Wesley, Reading, Mass, USA, 1989.

[10] B. Yousefi and F. Ershad, "Hypercyclic tuples and syndetic sequences," International Mathematical Forum, vol. 7, no. 9-12, pp. 579-584, 2012.

[11] G. Godefroy and J. H. Shapiro, "Operators with dense, invariant, cyclic vector manifolds," Journal of Functional Analysis, vol. 98, no. 2, pp. 229-269, 1991.

[12] S. Grivaux, "Hypercyclic operators, mixing operators, and the bounded steps problem," Journal of Operator Theory, vol. 54, no. 1, pp. 147-168, 2005.

[13] A. Peris and L. Saldivia, "Syndetically hypercyclic operators," Integral Equations and Operator Theory, vol. 51, no. 2, pp. 275281, 2005.

[14] R. Aron and D. Markose, "On universal functions," Journal of the Korean Mathematical Society, vol. 41, no. 1, pp. 65-76, 2004.

[15] N. S. Feldman, "Hypercyclic pairs of coanalytic Toeplitz operators," Integral Equations and Operator Theory, vol. 58, no. 2, pp. 153-173, 2007.

[16] J. A. Conejero and A. Peris, "Hypercyclic translation $C_{0}$ semigroups on complex sectors," Discrete and Continuous Dynamical Systems, Series A, vol. 25, no. 4, pp. 1195-1208, 2009. 


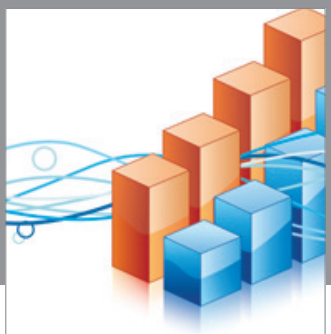

Advances in

Operations Research

vatem alat4

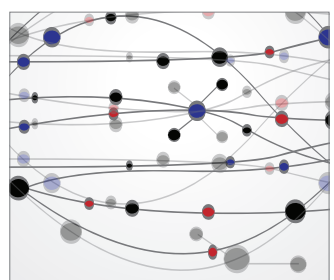

\section{The Scientific} World Journal
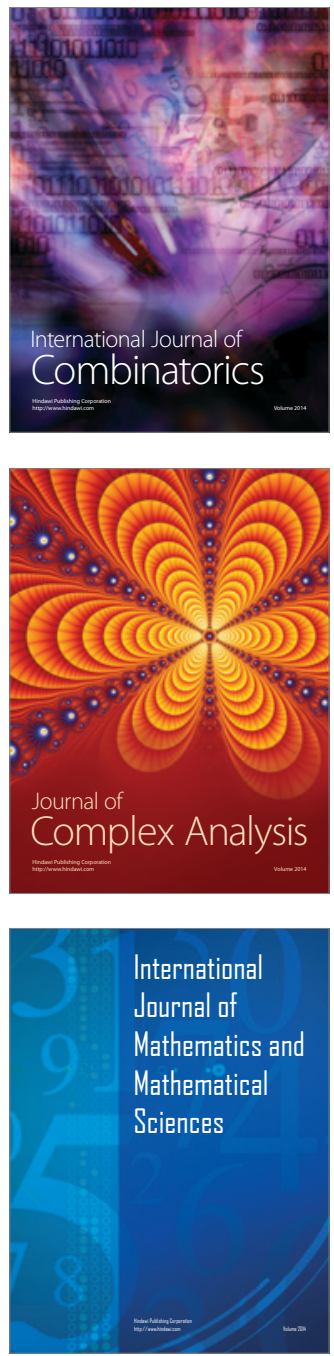
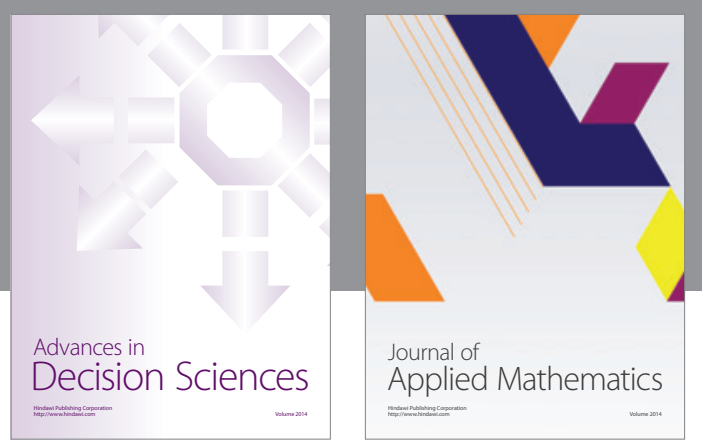

Algebra

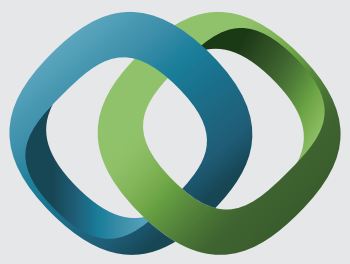

\section{Hindawi}

Submit your manuscripts at

http://www.hindawi.com
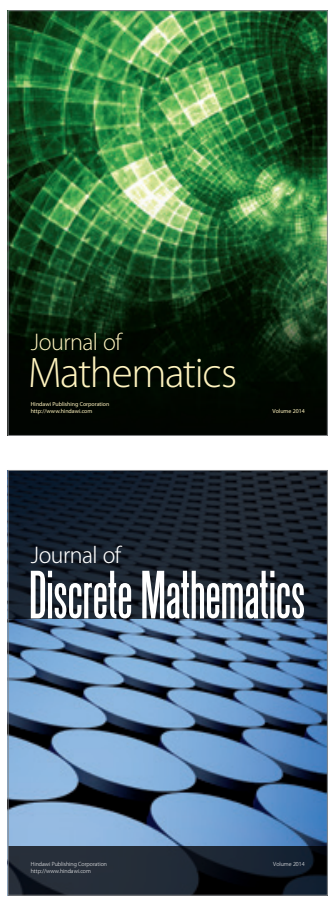

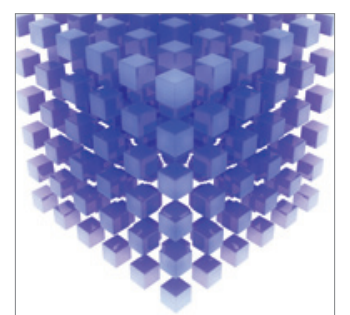

Mathematical Problems in Engineering
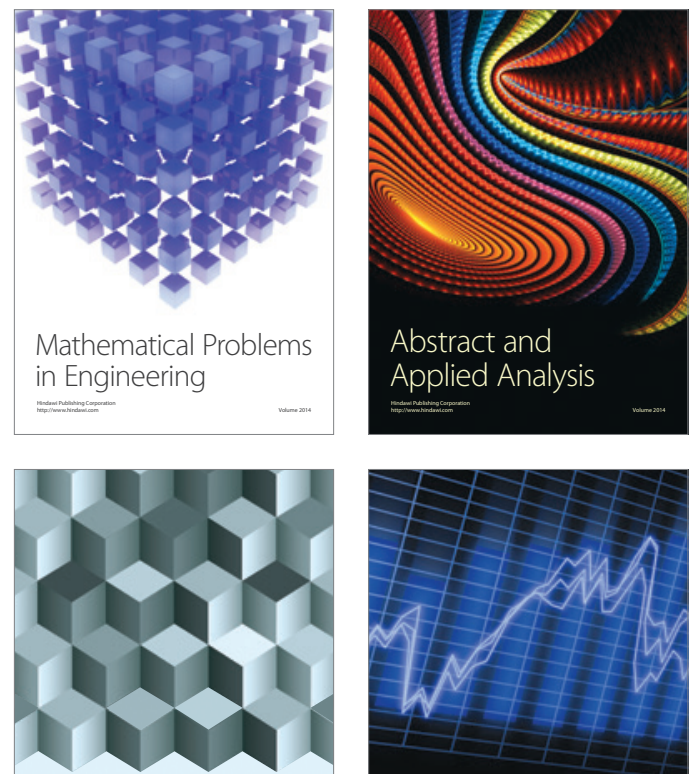

Journal of

Function Spaces

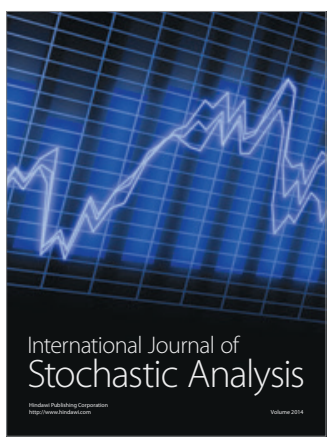

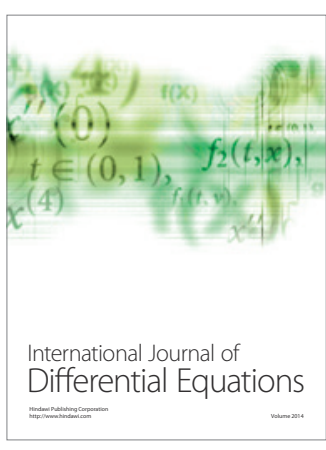
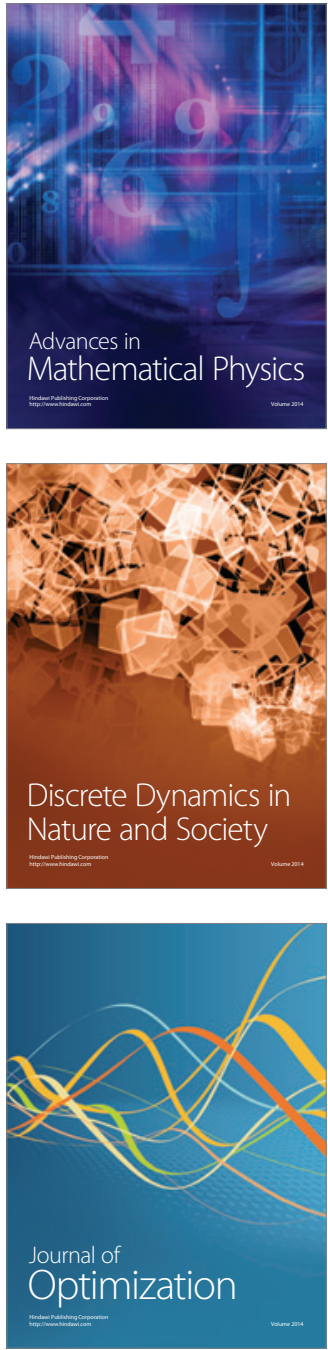